\title{
Relativistic Stellar Pulsations With Near-Zone Boundary Conditions*
}

\author{
Lee Lindblom \\ Theoretical Astrophysics 130-33, California Institute of Technology, Pasadena, CA 91125 \\ Gregory Mendell and James R. Ipser \\ Department of Physics, University of Florida, Gainesville, FL 32611
}

(August 17, 2021)

\begin{abstract}
A new method is presented here for evaluating approximately the pulsation modes of relativistic stellar models. This approximation relies on the fact that gravitational radiation influences these modes only on timescales that are much longer than the basic hydrodynamic timescale of the system. This makes it possible to impose the boundary conditions on the gravitational potentials at the surface of the star rather than in the asymptotic wave zone of the gravitational field. This approximation is tested here by predicting the frequencies of the outgoing non-radial hydrodynamic modes of non-rotating stars. The real parts of the frequencies are determined with an accuracy that is better than our knowledge of the exact frequencies (about 0.01\%) except in the most relativistic models where it decreases to about $0.1 \%$. The imaginary parts of the frequencies are determined with an accuracy of approximately $M / R$, where $M$ is the mass and $R$ is the radius of the star in question.
\end{abstract}

PACS Numbers: 04.40.Dg, 04.25.Nx, 95.30.Sf

\section{INTRODUCTION}

The pulsation of stars in general relativity theory has been the subject of scholarly investigation for many years. Consequently there are fairly well developed theories for modeling these oscillations [1] [2] [3] [4]. In comparison with their Newtonian counterparts, these stellar pulsations are fundamentally different, more interesting, and rather more difficult to evaluate. These differences arise because these pulsations couple to gravitational radiation. The illumination of a star by gravitational radiation, for example, causes that star to oscillate at the frequency of the incoming radiation. Thus, stars in general relativity theory can oscillate at any frequency at all!

The outgoing (or more commonly but less descriptively, the quasi-normal) modes of relativistic stars are defined by an additional boundary condition: that they oscillate without incoming gravitational radiation. This boundary condition is simple enough to understand; however, in practice it is rather difficult to impose numerically when finding the modes of realistic relativistic stellar models. This difficulty arises because the external gravitational perturbations must be decomposed into incoming and outgoing parts so that solutions having only outgoing radiation can be identified. This can only be done simply in the region far away from the star in the wave zone of the gravitational field. In non-rotating stars it is straightforward to integrate the exterior gravitational perturbations

*Published in Phys. Rev. D 56, 2118 (1997). out into the wave zone and perform this decomposition [2]. In that case the exterior gravitational field is the simple analytic Schwarzschild geometry, and the gravitational perturbations obey reasonably simple ordinary differential equations. In the case of rotating stars, however, this straightforward approach is not possible. The exterior geometries of rotating stars in general relativity are not known analytically. They are determined on numerical grids that become asymptotically poor as they approach the wave zone. The system of partial differential equations that determines the exterior gravitational perturbations of an oscillating star have never been solved numerically on such a grid. Thus, the study of the pulsations of rotating stars to date has been limited to the Newtonian [5] and post-Newtonian [6] approximations.

The purpose of this paper is to explore the possibility of replacing the outgoing-radiation boundary condition on the gravitational potentials with a condition that is easier to implement numerically. We derive conditions that are satisfied approximately by the outgoing gravitational perturbations in the near zone of the gravitational field. These conditions can easily be imposed on the gravitational fields in the well determined spacetime region just outside the star, even in numerically determined models. We propose that these near-zone boundary conditions be imposed on the gravitational potentials at the surface of the star as a substitute for the outgoingradiation boundary condition. We test the accuracy of this proposal by evaluating the non-radial modes of nonrotating stars, which can also be determined exactly. We find that the hydrodynamic modes are determined using the near-zone boundary condition with considerable precision: the real parts of the frequencies are determined with an accuracy better than our knowledge of the exact 
frequencies (about $0.01 \%$ ) except in the most relativistic models where it decreases to about $0.1 \%$. The imaginary parts are determined with an accuracy of about $M / R$ for typical neutron-star equations of state.

As part of this analysis we have derived a new representation of the exact relativistic pulsation equations for non-rotating stars, which is of considerable interest in its own right. We reduce the equations to a pair of secondorder ordinary differential equations for two scalar potentials: one fluid perturbation potential, and one gravitational potential. This representation of the equations is exactly analogous to the Newtonian pulsation equations [5]. The analysis of the near-zone boundary conditions described here is more straightforward using this new representation of the pulsation equations.

Section II describes the new representation of the relativistic pulsation equations in terms of two scalar potentials. Section III presents the standard boundary conditions used to determine the outgoing modes of relativistic stars in terms of the two-potential formalism introduced here. Section IV derives approximate expressions for the gravitational potentials of the outgoing modes that are valid in the near zone of the star. These expressions are used as the near-zone boundary conditions. The accuracy of these boundary conditions is tested by determining the modes of a family of typical neutron-star like relativistic stellar models. Section $\mathrm{V}$ concludes with a discussion of the feasibility of extending these near-zone techniques to rotating stars in general relativity theory. This paper also has two technical appendices which give in detail the new two-potential forms of the relativistic pulsation equations (Appendix A), and series solutions of these equations that are useful for imposing the boundary conditions within the star (Appendix B).

\section{NON-RADIAL PULSATIONS}

The pulsations of a relativistic stellar model are described by the perturbations in the spacetime metric $\delta g_{a b}$, and the associated perturbations in the stressenergy of the stellar matter $\delta T^{a b}$. The dynamical properties of these quantities are determined by the perturbed Einstein equation,

$$
\delta G^{a b}=8 \pi \delta T^{a b},
$$

and the appropriate outgoing gravitational radiation boundary condition at infinity. Here we consider in detail the non-radial pulsations of a spherical stellar model. It is convenient to describe the perturbations of the metric in such a spacetime using the Regge-Wheeler gauge, in which the metric and its perturbations have the following form,

$$
\begin{aligned}
& \left(g_{a b}+\delta g_{a b}\right) d x^{a} d x^{b}=-e^{\nu}\left(1+H_{0} Y_{l m} e^{i \omega t}\right) d t^{2} \\
& \quad+2 i H_{1} Y_{l m} e^{i \omega t} d t d r+e^{\lambda}\left(1-H_{0} Y_{l m} e^{i \omega t}\right) d r^{2} \\
& \quad+r^{2}\left(1-K Y_{l m} e^{i \omega t}\right)\left(d \theta^{2}+\sin ^{2} \theta d \varphi^{2}\right) .
\end{aligned}
$$

The perturbation functions $H_{0}, H_{1}$, and $K$, and the functions that describe the spacetime of the background equilibrium star, $\nu$ and $\lambda$, depend only on the radial coordinate $r$. The $Y_{l m}$ are the standard spherical harmonics, and the constant $\omega$ is the frequency of the mode. The explicit expressions for the components of the perturbed Einstein tensor $\delta G^{a b}$ that appear in Eq. (11) have been given numerous times elsewhere in terms of these fields [7], and will not be repeated here. We use standard geometrical units in which $G=c=1$.

The perturbations in the stress-energy tensor $\delta T^{a b}$ are assumed here to be those of a perfect fluid:

$$
\begin{aligned}
\delta T^{a b}= & (\delta \rho+\delta p) u^{a} u^{b}+(\rho+p)\left(\delta u^{a} u^{b}+u^{a} \delta u^{b}\right) \\
& +\delta p g^{a b}-p g^{a c} g^{b d} \delta g_{c d},
\end{aligned}
$$

where $\rho, p$, and $u^{a}$ denote the density, pressure, and four velocity $\left(u^{a} u_{a}=-1\right)$ of the unperturbed equilbrium model; and quantities preceeded by $\delta$ denote their Eulerian perturbations. For simplicity here we restrict our attention to simple barotropic perturbations, i.e., those where the density perturbation is proportional to the pressure perturbation:

$$
\delta \rho=\frac{d \rho}{d p} \delta p=\frac{d \rho}{d p} \delta p(r) Y_{l m} e^{i \omega t} .
$$

The perturbed conservation law $\delta\left(\nabla_{a} T^{a b}\right)=0$ can be solved analytically to obtain a simple and useful expression for the four-velocity perturbations. This solution is most conveniently expressed in terms of a scalar perturbation function $\delta U$, defined as

$$
\delta U(r)=\frac{\delta p(r)}{\rho+p}+\frac{1}{2} H_{0}(r) .
$$

Solving the spatial part of the perturbed conservation law gives an expression for $\delta u^{a}$ in terms of $\delta U$ and the metric perturbations [8]:

$$
\begin{aligned}
\delta u^{a} & =\frac{i}{\omega} e^{\nu / 2} e^{i \omega t} \nabla^{a}\left(\delta U Y_{l m}\right) \\
& +\left(\frac{1}{2} e^{\nu / 2} H_{0} \nabla^{a} t+e^{-\nu / 2} H_{1} \nabla^{a} r\right) Y_{l m} e^{i \omega t} .
\end{aligned}
$$

This expression allows us to describe a stellar oscillation completely in terms of the four scalar functions $\delta U, H_{0}$, $H_{1}$ and $K$.

The perturbed Einstein equation (11) can be used to reduce further the number of independent functions needed to determine the perturbed state of the star. The $\delta G^{r}{ }_{\theta}$ and $\delta G^{t}{ }_{\theta}$ components can be used to express $H_{1}$ and $H_{1}^{\prime}$ in terms of the other fields:

$$
\begin{gathered}
\omega e^{-\nu} H_{1}=H_{0}^{\prime}-K^{\prime}+\nu^{\prime} H_{0}, \\
\omega H_{1}^{\prime}=-\frac{1}{2} \omega\left(\nu^{\prime}-\lambda^{\prime}\right) H_{1}-\omega^{2} e^{\lambda}\left(H_{0}+K\right) \\
-16 \pi(\rho+p) e^{\nu+\lambda} \delta U .
\end{gathered}
$$

We use the notation ' to denote differentiation with respect to $r$. The $\delta G^{r}{ }_{r}$ and the $\delta G^{r}{ }_{t}$ components together 
with Eqs. (7) and (8) can similarly be used to express $K$ and $K^{\prime}$ in terms of $H_{0}, H_{0}^{\prime}, \delta U$ and $\delta U^{\prime}$ :

$$
\begin{gathered}
K=\alpha_{1} H_{0}^{\prime}+\alpha_{2} H_{0}+\alpha_{3} \delta U^{\prime}+\alpha_{4} \delta U, \\
K^{\prime}=\beta_{1} H_{0}^{\prime}+\beta_{2} H_{0}+\beta_{3} \delta U^{\prime}+\beta_{4} \delta U,
\end{gathered}
$$

where the functions $\alpha_{i}$ and $\beta_{i}$ depend on the equilibrium structure of the star (and the frequency) and are given explicitly in Appendix A. These expressions, Eqs. (7)(10), can be used to eliminate $H_{1}, H_{1}^{\prime}, K$ and $K^{\prime}$ from all of the remaining pulsation equations. The equations that determine the modes of relativistic stellar models can be reduced therefore to a pair of second-order equations for the fields $H_{0}$ and $\delta U$. A second-order equation for $H_{0}$ is obtained by using the derivative of Eq. (7) to eliminate $K^{\prime \prime}$ from the $\delta G^{t}{ }_{t}$ component of Einstein's equation. A second-order equation for $\delta U$ is obtained from the time component of the perturbed stress-energy conservation law. In each of these equations all occurances of $H_{1}$ and $H_{1}^{\prime}$ are eliminated with Eqs. (7) and (8), while $K$ and $K^{\prime}$ are eliminated using Eqs. (9) and (10). The resulting second-order equations can be expressed in the form:

$$
\begin{aligned}
& \delta U^{\prime \prime}+\left(\frac{2}{r}-\frac{\nu^{\prime}}{2} \frac{d \rho}{d p}+v_{1}\right) \delta U^{\prime} \\
& +\left[\frac{\omega^{2}}{e^{\nu}} \frac{d \rho}{d p}-\frac{l(l+1)}{r^{2}}+v_{2}\right] e^{\lambda} \delta U \\
& =v_{3} H_{0}^{\prime}+\left[\frac{\omega^{2}}{2 e^{\nu}} \frac{d \rho}{d p}+v_{4}\right] e^{\lambda} H_{0}, \\
& H_{0}^{\prime \prime}+\left(\frac{2}{r}+\eta_{1}\right) H_{0}^{\prime} \\
& +\left[\frac{\omega^{2}}{e^{\nu}}-\frac{l(l+1)}{r^{2}}+4 \pi(\rho+p) \frac{d \rho}{d p}+\eta_{2}\right] e^{\lambda} H_{0} \\
& =\eta_{3} \delta U^{\prime}+\left[8 \pi(\rho+p) \frac{d \rho}{d p}+\eta_{4}\right] e^{\lambda} \delta U,
\end{aligned}
$$

where the functions $v_{i}$ and $\eta_{i}$ depend on the structure of the equilibrium star (and the frequency) and are given in Appendix A.

Equations (11) and (12) are the general equations for the linear (even-parity) modes of general relativistic stellar models. This form of the equations has several advantages over earlier representations of the fourth-order system needed to describe these oscillations [2], [9], [10]. Equations (11) and (12) reduce in a straightforward way, for example, to the Newtonian equations for the nonradial modes in the appropriate weak-field slow-motion limit. (Simply set $v_{i}=\eta_{i}=0, \nu^{\prime}=-2 p^{\prime} / \rho, e^{\nu}=e^{\lambda}=1$ in Eqs. [11] and [12], and $\omega^{2}=p=0$ in Eq. [12].) The equation for the gravitational perturbation $H_{0}$ reduces to a scalar wave equation in the region of spacetime far away from the star, and in the static limit to Laplace's equation. Thus it is straightforward to determine the asymptotic behavior of the perturbed fields from this representation of the equations. The functions $v_{i}$ and $\eta_{i}$ are smaller than the terms explicitly given in Eqs. (11) and (12) by a factor of order $M / R$, with $M$ the total mass and $R$ the total radius of the star. Thus these terms are small except in the interiors of extremely relativistic models. A simple and elegant form of the relativistic Cowling approximation [8] 11] can be obtained by setting $H_{0}=0$ in Eq. (11). In this case the single fluid potential $\delta U$ is determined by a single second-order wave equation. The solutions to this equation give fairly good approximations of the hydrodynamic modes of relativistic stars [11]. Similarly we presume that approximate expressions for the even-parity $w$-modes (or 'wave' modes [4]) could be obtained by setting $\delta U=0$ in Eq. (12) and solving the resulting homogeneous second-order equation for $H_{0}$.

\section{BOUNDARY CONDITIONS}

The physical solutions to Eqs. (11) and (12) are identified by imposing suitable boundary conditions at the center and surface of the fluid interior of the star, and also in the asymptotic wave zone of the gravitational field. The boundary conditions associated with the stellar fluid region are rather simple. At the center of the star the perturbations are simply required to be finite. This condition eliminates one singular solution to the equations for each of the functions $H_{0}$ and $\delta U$. The non-singular solutions can be approximated as power series near $r=0$,

$$
\begin{aligned}
& \delta U=A r^{l}+\mathcal{O}\left(r^{l+2}\right), \\
& H_{0}=B r^{l}+\mathcal{O}\left(r^{l+2}\right),
\end{aligned}
$$

where $A$ and $B$ are arbitrary constants.

At the surface of the fluid region a boundary condition must also be imposed that fixes the location of the perturbed surface of the star. Those thermodynamic potentials that vanish continuously on the surface of the equilibrium star (e.g. the pressure) must also vanish on the moving surface of the perturbed star. This condition is equivalent to the requirement that the Lagrangian perturbation of these potentials must vanish at the surface of the star [12]. The thermodynamic potential $h(p)$ defined by

$$
h(p)=\int_{0}^{p} \frac{d p^{\prime}}{\rho\left(p^{\prime}\right)+p^{\prime}}
$$

is the ideal choice to implement this boundary condition 13. The needed condition is traditionally written $\Delta h=$ 0 on the surface of the star. This condition can also be re-expressed in terms of the Eulerian quantities as

$$
\Delta h=\delta h+\frac{e^{\nu / 2}}{i \omega} \delta u^{a} \nabla_{a} h=0,
$$


for the perturbations of a non-rotating star considered here. Since $\delta h=\delta p /(\rho+p)$, this expression can be rewritten in terms of the fields $\delta U$ and $H_{0}$ :

$$
\begin{aligned}
& \omega^{2} e^{\lambda-\nu}\left(\delta U-\frac{1}{2} H_{0}\right) \\
& -\frac{1}{2} \nu^{\prime}\left[\delta U^{\prime}-\left(1-\beta_{1}\right) H_{0}^{\prime}-\left(\nu^{\prime}-\beta_{2}\right) H_{0}\right]=0 .
\end{aligned}
$$

In Appendix B power series expressions (valid near the surface of the star) are presented for the fields $H_{0}$ and $\delta U$ which satisfy this condition. These series solutions are useful for imposing the boundary condition numerically on a finite grid having points near, but not on the actual surface of the star.

The boundary conditions associated with the fluid interior of the star, Eqs. (13), (14), and (17), are sufficient to fix a unique (up to overall scale) solution to Eqs. (11) and (12) for each value of the frequency $\omega$. These conditions do not however fix the frequency of the pulsation modes of these stars. Indeed, relativistic stars can oscillate at any frequency at all if they are driven by incoming gravitational radiation of that frequency. The outgoing modes of relativistic stars are defined by the additional condition that they contain no incoming gravitational radiation. This condition must be imposed as an additional boundary condition on the gravitational potentials in the asymptotic wave zone.

In the exterior of the star the gravitational potential $H_{0}$ is determined by the vacuum $\left(\rho=p=\eta_{3}=\eta_{4}=0\right)$ limit of Eq. (12). The behavior of the solutions in the asymptotic wave zone are of particular interest to us. Thus, we consider the limiting form of this equation when $M / r \ll 1$, where $M$ is the total mass of the star. In this limit Eq. (12) reduces to the homogeneous equation,

$$
H_{0}^{\prime \prime}+\left(\frac{2}{r}+\eta_{1}\right) H_{0}^{\prime}+\left[\omega^{2}-\frac{l(l+1)}{r^{2}}+\eta_{2}\right] H_{0}=0
$$

where $\eta_{1}$ and $\eta_{2}$ are given in this case by

$$
\begin{gathered}
\eta_{1}=\frac{4}{r}\left\{(l-1)(l+2) \Delta\left[l(l+1)-2 r^{2} \omega^{2}\right]-1\right\}, \\
\eta_{2}=16 r^{2} \omega^{4} \Delta,
\end{gathered}
$$

and where $\Delta$ reduces to

$$
\frac{1}{\Delta}=\left[(l-1)(l+2)-2 r^{2} \omega^{2}\right]\left[l(l+1)-2 r^{2} \omega^{2}\right]+4 r^{2} \omega^{2} .
$$

Remarkably, the general solution to this equation has been found analytically [14:

$$
\begin{array}{r}
H_{0}=-\left[r \omega \frac{d}{d(r \omega)}+1+\frac{1}{2} l(l+1)-(r \omega)^{2}\right] \\
\times \frac{2 i \omega^{l+1}\left[C j_{l}(r \omega)+D y_{l}(r \omega)\right]}{l(l-1)(2 l-1) ! !},
\end{array}
$$

where $j_{l}$ and $y_{l}$ are spherical Bessel functions and $C$ and $D$ are arbitrary constants. In the asymptotic wave zone, $r \omega \gg 1$, the Bessel functions have simple asymptotic forms:

$$
\begin{aligned}
& {\left[C j_{l}(r \omega)+D y_{l}(r \omega)\right] e^{i \omega t}} \\
& \quad=\frac{D+i C}{2 r \omega} e^{i \omega(t+r)-i l \pi / 2}+\frac{D-i C}{2 r \omega} e^{i \omega(t-r)+i l \pi / 2} \\
& \quad+\mathcal{O}(r \omega)^{-2}
\end{aligned}
$$

The condition that the solution contain no incoming radiation reduces to the constraint $D=-i C$. Thus the required boundary condition on the gravitational potential $H_{0}$ is that it approach (as $r \rightarrow \infty$ ) the asymptotic expression

$$
H_{0}=-\frac{2 C r \omega^{l+2} e^{-i \omega r+i l \pi / 2}}{l(l-1)(2 l-1) ! !}\left[1+\mathcal{O}(r \omega)^{-1}\right],
$$

where $C$ is an arbitrary constant.

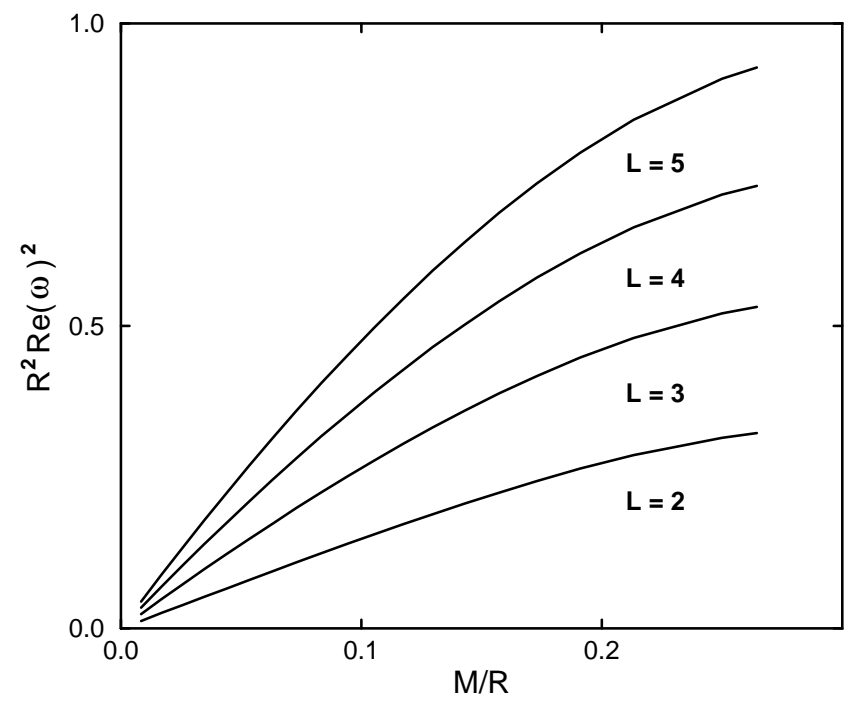

FIG. 1. Frequencies of the fundamental hydrodynamic modes as a function of $M / R$ for typical relativisitic stellar models.

\section{NEAR-ZONE BOUNDARY CONDITIONS}

The frequencies of the hydrodynamic modes of interest to us here are approximately related to the mass $M$ and radius $R$ of the star by $R^{2} \omega^{2} \approx l M / R$. Figure 1 illustrates the frequencies of these modes for "typical" relativistic stellar models computed from the simplified neutron-star like equation of state $p=\kappa \rho^{2}$, with $\kappa=1.3346 \times 10^{5} \mathrm{~cm}^{5} / \mathrm{g} \mathrm{s}^{2}$. (The maximum mass model for this equation of state has $M=1.911 M_{\odot}$, and the radius of the $1.4 M_{\odot}$ model is $R=14.45 \mathrm{~km}$.) The surfaces of these stars are approximately in the near zone of the gravitational potential, $r^{2} \omega^{2}<1$, and also in the zone (an extremely good approximation for the more Newtonian models) where $M / r \ll 1$. The expression for $H_{0}$ 
given in Eq. (22) is valid in the entire exterior region of the star where $M / r \ll 1$. Thus, it is also valid in a portion of the near zone for these modes. In this region the expression for $H_{0}$ can be simplified by employing the asymptotic expansions for the spherical Bessel functions in the limit $r^{2} \omega^{2} \ll 1$ :

$H_{0}=\frac{C}{r^{l+1}}\left\{1-i N_{l}(r \omega)^{2 l+1}+\mathcal{O}(r \omega)^{2}+\mathcal{O}(M / r)\right\}$,

where $N_{l}$ is given by

$$
N_{l}=\frac{(l+1)(l+2)}{l(l-1)(2 l+1)[(2 l-1) ! !]^{2}} .
$$

The lowest-order version of the near-zone boundary condition sets $H_{0}$ to the value given by Eq. (25):

$$
H_{0}=\frac{C}{r^{l+1}}\left[1-i N_{l}(r \omega)^{2 l+1}\right] .
$$

The value of the derivative $H_{0}^{\prime}$ is obtained by differentiation of Eq. (27). The next question is, "Where should these conditions be imposed?" Moving the boundary away from the star improves the accuracy of the condition $M / r \ll 1$ used to derive Eq. (25), but reduces the accuracy of the condition $r^{2} \omega^{2} \ll 1$. As Fig. 1 illustrates, the $r^{2} \omega^{2}<1$ condition is just barely satisfied on the surfaces of the most relativistic stellar models. Thus, the most appropriate place to impose the near-zone boundary condition, Eq. (27), is directly on the surface of the star. We have solved the relativistic pulsation equations (11)-(12) numerically using these lowest-order near-zone boundary conditions for the stellar models described above. Figures 2 and 3 illustrate the real and imaginary parts of the frequencies determined in this way. The frequencies in these figures are given in units of $\sqrt{M / R^{3}}$. Also depicted in these figures are the frequencies obtained by solving Eqs. (11)-(12) with the exact outgoing-radiation boundary condition, Eq. (24), imposed far away from the star in the wave zone. These figures illustrate that the near-zone boundary condition does reproduce in a qualitative way the frequencies of the hydrodynamic modes of these stars. For the more Newtonian models, $M / R \ll 1$, the frequencies are determined quite accurately (as expected). For the more relativistic models, however, the agreement is not as good: the real parts of the frequencies agree with the exact to within about $4 \%$, while the imaginary parts only agree to within about an order of magnitude. This level of accuracy is, nevertheless, somewhat better than that obtained with the standard postNewtonian approximation [6].

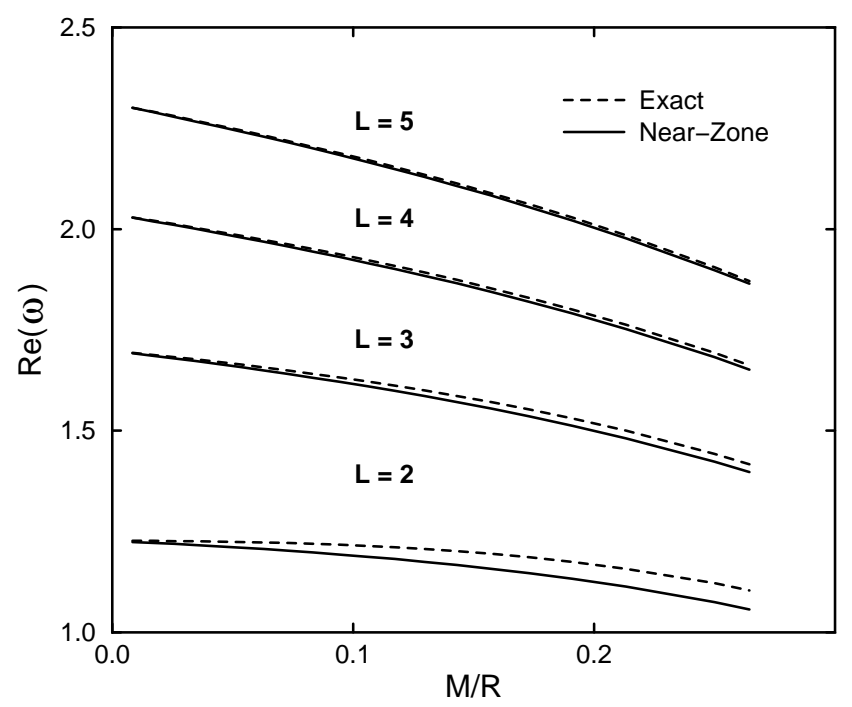

FIG. 2. Real parts of the frequencies computed with the lowest-order near-zone boundary condition as given in Eq. (27).

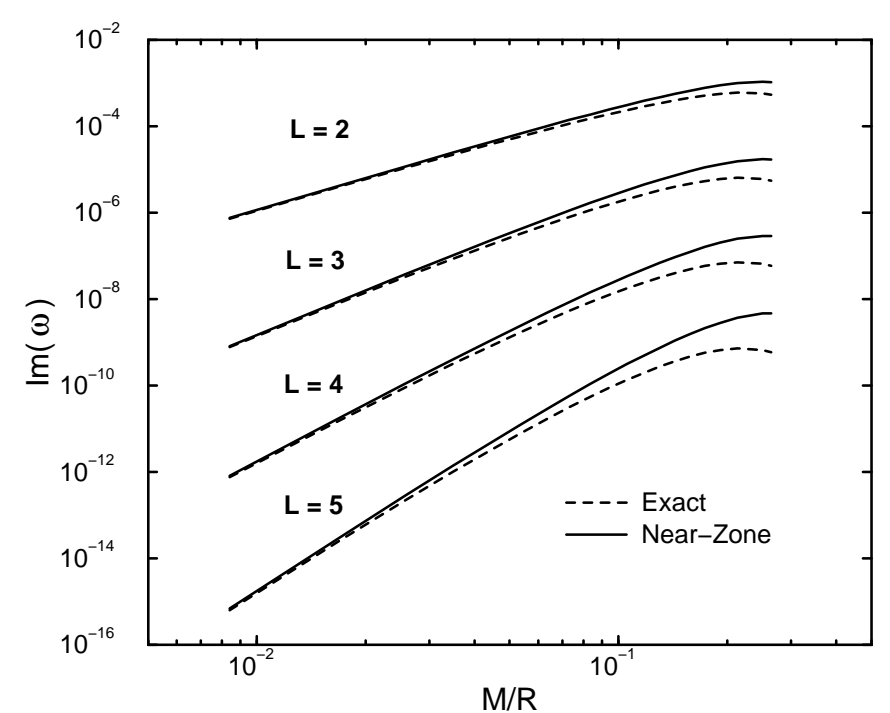

FIG. 3. Imaginary parts of the frequencies computed with the lowest-order near-zone boundary condition as given in Eq. 27).

The expression for $H_{0}$ given in Eq. (25) includes only the lowest-order terms in $(r \omega)^{2}$ and $M / r$. By keeping additional terms we could in principle construct a sequence of higher-order (and one might expect more accurate) near-zone boundary conditions. We have found (after considerable numerical experimentation) that the real part of $H_{0}$ is most sensitive to the $M / r$ terms in this expansion, while the imaginary part is most sensitive to the $(r \omega)^{2}$ terms. This makes a certain amount of physical sense: the real part of $H_{0}$ most strongly influences the real part of $\omega$. This hydrodynamic aspect of the mode is most sensitive to the static "Newtonian" $M / r$ behavior of the potential. The imaginary part of $H_{0}$ determines, in effect, the imaginary part of $\omega$. This 
radiation-reaction aspect of the mode is most sensitive to the dynamical $(r \omega)^{2}$ behavior of the potential. Thus, we have constructed a boundary condition that keeps the $M / r$ terms to all orders for the real part of $H_{0}$, while keeping the $(r \omega)^{2}$ terms to all orders for the imaginary part of $H_{0}$ :

$$
\begin{aligned}
H_{0}= & C\left\{Q^{2}{ }_{l}(x)\left[1+\frac{(l-8)(r \omega)^{2}}{2 l(2 l-1)}\right]\right. \\
& \left.-i K_{l}\left[r \omega \frac{d}{d(r \omega)}+1+\frac{1}{2} l(l+1)-(r \omega)^{2}\right] j_{l}(r \omega)\right\},
\end{aligned}
$$

where $C$ is an arbitrary constant, $x=r / M-1$, and $K_{l}$ is given by

$$
K_{l}=\frac{2(l+2) !(M \omega)^{l+1}}{l(l-1)(2 l-1) ! !(2 l+1) ! !} .
$$

The function $Q_{l}^{2}(x)$ is the associated Legendre function of the second kind. It is the exact solution to the static $(\omega=0)$ limit of the $H_{0}$ equation, Eq. (12), that falls off like $1 / r^{l+1}$ for large values of $r$ 15. Thus, the real part of $H_{0}$ is given in Eq. (28) by an expression that is exact to all orders in $M / r$ when $\omega=0$. The lowest-order $(r \omega)^{2}$ correction has also been added to this term. The imaginary part of $H_{0}$ in Eq. (28) is taken to be the exact solution to the $H_{0}$ equation (12) when $M=0$. This is just the spherical Bessel function solution that is given in Eq. (22) with the outgoing-radiation boundary condition. This solution is exact to all orders in $(r \omega)^{2}$ when $M=0$. The constant $K_{l}$ was chosen so that the expression in Eq. (28) approaches (27) in the appropriate $M / r \ll 1$ and $(r \omega)^{2} \ll 1$ limit.

We have solved the relativistic pulsation equations (11)-(12) numerically using the higher-order near-zone boundary condition given in Eq. (28) imposed at the surface of the star $r=R$. The derivative of $H_{0}$ is set to the derivative of Eq. (28) at $r=R$. Figures 4 and 5 illustrate the real and imaginary parts of the frequencies computed in this way. The real parts of the frequencies computed with the higher-order near-zone boundary conditions agree with those computed with the exact outgoing-radiation boundary condition with impressive accuracy. The fractional error is less than about $0.01 \%$, the numerical accuracy with which we compute the exact frequencies, except in the most relativistic models. For the $l=2 f$-modes this error is about $0.1 \%$ in the maximum mass model, and about $0.02 \%$ for the $1.4 M_{\odot}$ model. The accuracy of the real parts of the frequencies increases as $l$ increases. The imaginary parts of the frequencies are determined with accuracy of about $M / R$; considerably better than was obtained with the lowest-order near-zone boundary condition. The maximum error is about $33 \%$ for the $l=3$ mode of the most relativistic model. The accuracy for the modes of the $1.4 M_{\odot}$ models is about $10 \%$. This level of accuracy in the imaginary part of the frequency is consistent with the fact that we have ignored terms of order $M / r$ in Eq. (28) [17].

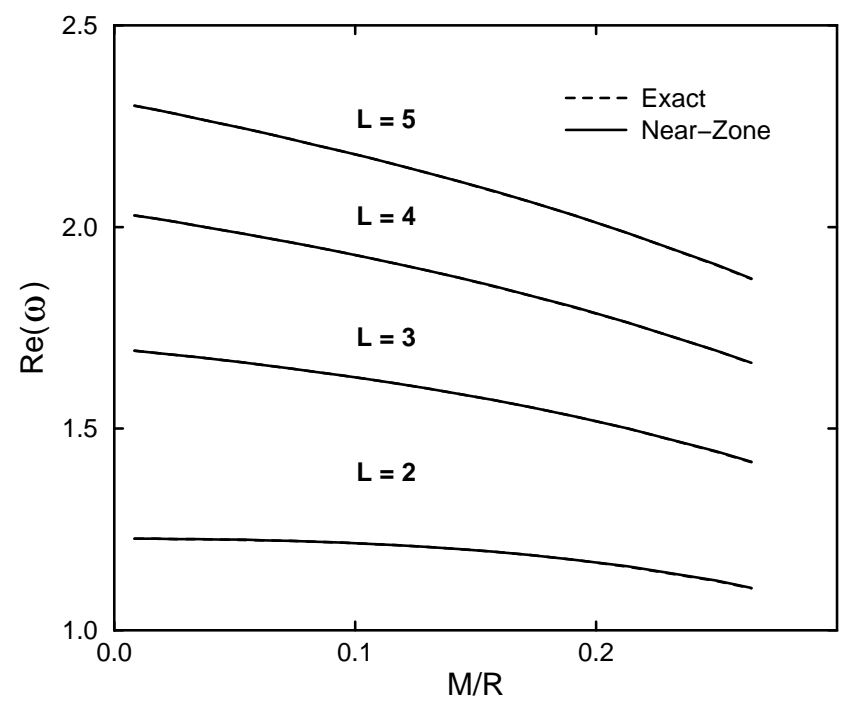

FIG. 4. Real parts of the frequencies computed with the higher-order near-zone boundary condition as given in Eq. (28).

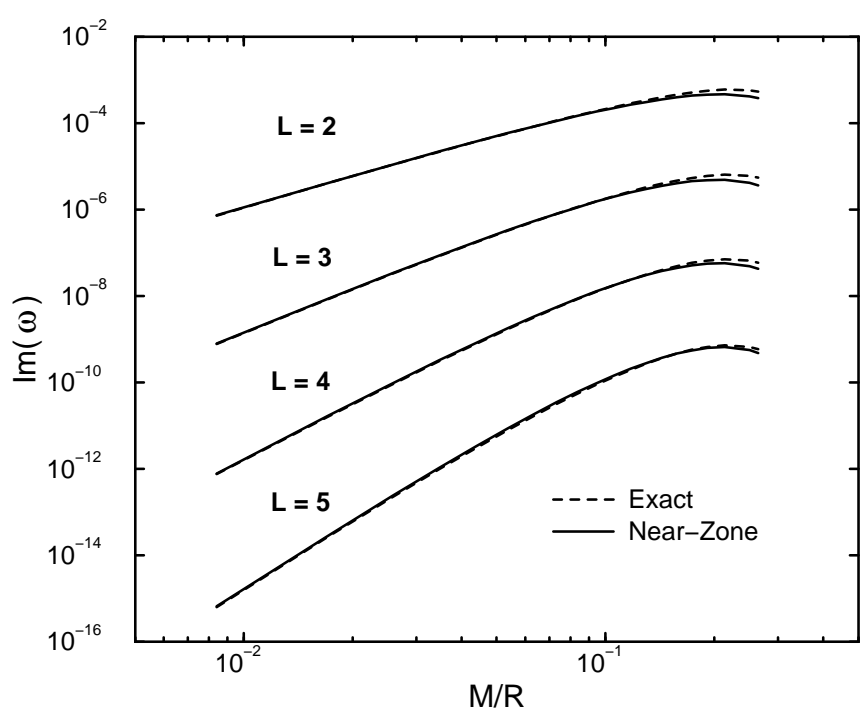

FIG. 5. Imaginary parts of the frequencies computed with the higher-order near-zone boundary condition as given in Eq. (28).

To further illustrate the accuracy of the near-zone boundary conditions we present in Table 1 the frequencies for the modes of the $1.4 M_{\odot}$ models computed with several different approximation methods. The Cowling approximation used here is obtained by setting $H_{0}=0$ in Eq. (11). The post-Newtonian frequencies presented here are obtained from the $\gamma=2$ post-Newtonian frequencies tabulated by Cutler and Lindblom [6] and adjusted to the particular model considered here. The near-zone boundary conditions were used in both the lowest-order form 
of Eq. (27) and the higher-order form of Eq. 28).

The detailed numerical results presented here were obtained for the $\gamma=2$ polytropic equation of state described above. We have also evaluated the modes of neutron star models based on a softer $\gamma=5 / 3$ polytropic equation of state, and also on the more realistic BetheJohnson equation of state 18. We find that the accuracy of the frequencies in these two additional cases is comparable to (and in fact slightly better than) the $\gamma=2$ polytropic case discussed in detail here.

\section{CONCLUDING REMARKS}

This paper presents a new formulation of the relativistic pulsation equations in terms of two scalar potentials: one fluid and one gravitational perturbation field. These potentials satisfy a pair of second-order ordinary differential equations that are analogous to the Newtonian pulsation equations. These equations are quite general: they describe the complete set of even-parity modes of relativistic stars, including the $w$-modes. This formulation of the relativistic pulsation equations has a significantly simpler structure than earlier representations.

The primary purpose of this paper was to explore the possibility of replacing the outgoing-radiation boundary condition imposed in the wave zone with appropriate conditions imposed on the surface of the star. We have used the two-potential forms for the stellar pulsation equations to derive the appropriate near-zone boundary conditions on the gravitational potential. We have shown that the outgoing hydrodynamic modes can be determined with considerable precision using these near-zone boundary conditions. Unfortunately this success is somewhat vacuous: we already knew how to evaluate these modes exactly. However we believe that this proceedure should also allow us to evaluate the modes of rapidly rotating fully relativistic stars with a precision similar to that obtained here. The near-zone boundary conditions that we present here depend only weakly on the structure of the exterior gravitational field of the star. The boundary condition used to determine the real part of the potential $H_{0}$ does depend on the exact static solution to the exterior gravitational perturbation equations. But such static solutions have been found numerically in rapidly-rotating strongly relativistic models [16]. We believe that these numerical solutions can be used to formulate a near-zone boundary condition that will be accurate even for rapidly rotating models. The boundary condition on the imaginary part of $H_{0}$ does not depend on the structure of the external gravitational field of the star at all: it is just the flat-space perturbation representing an outgoing wave. Finally, the modes of primary interest in rotating stars have the property that their frequencies $\omega$ decrease toward zero as the angular velocity of the star increases. For these modes the near-zone approximation $r^{2} \omega^{2} \ll 1$ on which our boundary condition is based will be satisfied more exactly as the angular velocity of the star increases.

\section{ACKNOWLEDGMENTS}

We thank C. Cutler, S. Detweiler, J. Friedman, B. Schutz, and K. Thorne for helpful discussions concerning this work. This research was supported by NSF grants PHY-9796079 and PHY-9408910, NASA grants NAG54093 and NAG5-3936, and the Institute for Fundamental Theory, University of Florida. L.L. thanks the Max Plank Institute für Gravitationsphysik, Potsdam and the Institute for Fundamental Theory, University of Florida for their hospitality during visits in which portions of this research were completed. 


\section{APPENDIX A: THE PULSATION EQUATIONS} $\delta U^{\prime}$ :

In Section $\amalg$ are presented the general forms of the equations that express $K$ and $K^{\prime}$ in terms of $H_{0}, H_{0}^{\prime}, \delta U$ and

$$
\begin{aligned}
K & =\alpha_{1} H_{0}^{\prime}+\alpha_{2} H_{0}+\alpha_{3} \delta U^{\prime}+\alpha_{4} \delta U, \\
K^{\prime} & =\beta_{1} H_{0}^{\prime}+\beta_{2} H_{0}+\beta_{3} \delta U^{\prime}+\beta_{4} \delta U .
\end{aligned}
$$

Here we present the complete expressions for the coefficients $\alpha_{i}$ and $\beta_{i}$ :

$$
\begin{aligned}
& \alpha_{1}=-r \Delta\left\{4 r^{2} \omega^{2} e^{-\nu}+r \nu^{\prime}\left[16 \pi r^{2}(\rho+p)-l(l+1)\right]\right\}, \\
& \alpha_{2}= \Delta\left\{r\left(2-r \nu^{\prime}\right)\left[16 \pi r^{2}(\rho+p) \nu^{\prime}-l(l+1) \nu^{\prime}+2 r \omega^{2} e^{-\nu}\right]\right. \\
&\left.-\left[l(l+1) e^{\lambda}-2\left(1-r \nu^{\prime}\right)-8 \pi r^{2}(\rho+p) e^{\lambda}\right]\left[16 \pi r^{2}(\rho+p)-l(l+1)+2 r^{2} \omega^{2} e^{-\nu}\right]\right\}, \\
& \alpha_{3}=-16 \pi r^{3} \Delta(\rho+p)\left(2-r \nu^{\prime}\right), \\
& \alpha_{4}=-16 \pi r^{2}(\rho+p) e^{\lambda} \Delta\left[16 \pi r^{2}(\rho+p)-l(l+1)+2 r^{2} \omega^{2} e^{-\nu}\right], \\
& \beta_{1}=\Delta\left\{\left[2 r^{2} \omega^{2} e^{-\nu}-(l-1)(l+2)\right]\left[16 \pi r^{2}(\rho+p)-l(l+1)\right] e^{\lambda}+2 r^{2} \omega^{2} e^{-\nu}\left(2-r \nu^{\prime}\right)\right\}, \\
& \beta_{2}=\Delta\left\{r \omega^{2} e^{-\nu}\left(2-r \nu^{\prime}\right)\left[l(l+1) e^{\lambda}-2\left(1-r \nu^{\prime}\right)-8 \pi r^{2}(\rho+p) e^{\lambda}\right]\right. \\
&\left.\quad \quad-\left[(l-1)(l+2)-2 r^{2} \omega^{2} e^{-\nu}\right]\left[16 \pi r^{2} \nu^{\prime}(\rho+p)-l(l+1) \nu^{\prime}+2 r \omega^{2} e^{-\nu}\right] e^{\lambda}\right\}, \\
& \beta_{3}=16 \pi r^{2}(\rho+p) \Delta e^{\lambda}\left[(l-1)(l+2)-2 r^{2} \omega^{2} e^{-\nu}\right], \\
& \beta_{4}=16 \pi r^{3} \omega^{2}\left(2-r \nu^{\prime}\right)(\rho+p) \Delta e^{\lambda-\nu},
\end{aligned}
$$

where $\Delta$ is defined by

$$
\Delta^{-1}=\left[(l-1)(l+2)-2 r^{2} \omega^{2} e^{-\nu}\right]\left[l(l+1)-2 r^{2} \omega^{2} e^{-\nu}-16 \pi r^{2}(\rho+p)\right] e^{\lambda}+r^{2} \omega^{2} e^{-\nu}\left(2-r \nu^{\prime}\right)^{2} .
$$

This transformation becomes singular whenever $\Delta^{-1}$ vanishes. We do not yet understand the general conditions under which this singularity can occur (if any). We have determined numerically, however, that $\Delta>0$ for all $r$ in the hydrodynamic modes studied here.

Also in Section II were presented the general forms of the second-order equations for $\delta U$ and $H_{0}$ :

$$
\begin{aligned}
& \delta U^{\prime \prime}+\left(\frac{2}{r}-\frac{\nu^{\prime}}{2} \frac{d \rho}{d p}+v_{1}\right) \delta U^{\prime}+\left[\frac{\omega^{2}}{e^{\nu}} \frac{d \rho}{d p}-\frac{l(l+1)}{r^{2}}+v_{2}\right] e^{\lambda} \delta U=v_{3} H_{0}^{\prime}+\left[\frac{\omega^{2}}{2 e^{\nu}} \frac{d \rho}{d p}+v_{4}\right] e^{\lambda} H_{0} \\
& H_{0}^{\prime \prime}+\left(\frac{2}{r}+\eta_{1}\right) H_{0}^{\prime}+\left[\frac{\omega^{2}}{e^{\nu}}-\frac{l(l+1)}{r^{2}}+4 \pi(\rho+p) \frac{d \rho}{d p}+\eta_{2}\right] e^{\lambda} H_{0} \\
&=\eta_{3} \delta U^{\prime}+\left[8 \pi(\rho+p) \frac{d \rho}{d p}+\eta_{4}\right] e^{\lambda} \delta U
\end{aligned}
$$

Here we give the complete expressions for the coefficients $v_{i}$ and $\eta_{i}$ :

$$
\begin{aligned}
& v_{1}=\nu^{\prime}-\frac{1}{2} \lambda^{\prime}+\left[\frac{2}{r}-\frac{\nu^{\prime}}{2}\left(\frac{d \rho}{d p}+1\right)\right] \beta_{3}, \\
& v_{2}=16 \pi(\rho+p)+e^{-\lambda}\left[\frac{2}{r}-\frac{\nu^{\prime}}{2}\left(\frac{d \rho}{d p}+1\right)\right] \beta_{4}, \\
& v_{3}=\left[\frac{2}{r}-\frac{\nu^{\prime}}{2}\left(\frac{d \rho}{d p}+1\right)\right]\left(1-\beta_{1}\right), \\
& v_{4}=-\frac{1}{2} \omega^{2} e^{-\nu}+e^{-\lambda}\left[\frac{2}{r}-\frac{\nu^{\prime}}{2}\left(\frac{d \rho}{d p}+1\right)\right]\left(\nu^{\prime}-\beta_{2}\right), \\
& \eta_{1}=\frac{1}{2}\left(\nu^{\prime}-\lambda^{\prime}\right)+\frac{2}{r}\left(2-r \nu^{\prime}\right)\left(\beta_{1}-1\right), \\
& \eta_{2}=e^{-\lambda}\left\{\nu^{\prime \prime}+\left(\nu^{\prime}\right)^{2}+\frac{1}{2 r}\left(2-r \nu^{\prime}\right)\left[4 \beta_{2}-\nu^{\prime}+\lambda^{\prime}\right]\right\}+4 \pi(\rho+p), \\
& \eta_{3}=-\frac{2}{r}\left(2-r \nu^{\prime}\right) \beta_{3}, \\
& \eta_{4}=-8 \pi(\rho+p)-\frac{2}{r}\left(2-r \nu^{\prime}\right) e^{-\lambda} \beta_{4} .
\end{aligned}
$$




\section{APPENDIX B: POWER SERIES SOLUTIONS}

It is helpful to have power series solutions to Eqs. (11) and (12) for the functions $\delta U$ and $H_{0}$ that satisfy the appropriate boundary conditions near the center, $r=0$, and near the surface, $r=R$, of the star. Such series solutions are useful, for example, in allowing the boundary conditions to be imposed numerically at grid points near, but not on the actual boundaries. Near $r=0$ the situation is straightforward: the functions $\delta U$ and $H_{0}$ are given by the series solutions

$$
\begin{aligned}
& \delta U=A r^{l}+\mathcal{O}\left(r^{l+2}\right), \\
& H_{0}=B r^{l}+\mathcal{O}\left(r^{l+2}\right),
\end{aligned}
$$

where $A$ and $B$ are arbitrary constants.

Near the surface of the star, however, the situation is more delicate. The thermodynamic derivative $d \rho / d p$ typically diverges at the surface of the star. Thus the second derivatives of $\delta U$ and $H_{0}$ may be infinite at $r=$ $R$. This makes a straightforward power series expansion impossible. For definiteness we assume here that in the neighborhood of the surface of the star the equation of state can be represented as a polytrope

$$
p=\kappa \rho^{\gamma},
$$

where $\kappa$ and $\gamma$ are constants. Near the surface of such a star the equilibrium structure equations can be solved as power series expansions. From this we learn that

$$
\begin{gathered}
\frac{d \rho}{d p}=\frac{\rho}{\gamma p}=\frac{R(R-2 M)}{(\gamma-1) M(R-r)}[1+\mathcal{O}(R-r)], \\
\rho=\left[\frac{\gamma-1}{\gamma \kappa} \frac{M(R-r)}{R(R-2 M)}\right]^{\frac{1}{\gamma-1}}[1+\mathcal{O}(R-r)] .
\end{gathered}
$$

Thus the derivative $d \rho / d p$ that appears as a coefficient in Eq. (11) always diverges for polytropes. The combination $(\rho+p) d \rho / d p$ that appears in Eq. (12) diverges for stiff equations of state $(\gamma>2)$ but not for softer equations of state:

$$
(\rho+p) \frac{d \rho}{d p}=\frac{1}{\kappa \gamma}\left[\frac{\gamma-1}{\gamma \kappa} \frac{M(R-r)}{R(R-2 M)}\right]^{\frac{2-\gamma}{\gamma-1}}[1+\mathcal{O}(R-r)] .
$$

The boundary conditions determine the surface values of $\delta U^{\prime}(R)$ and $H_{0}^{\prime}(R)$. The value of $H_{0}^{\prime}(R)$ is determined by continuity at $r=R$ from the outgoing solution in the exterior of the star. The value of $\delta U^{\prime}(R)$ is determined by the boundary condition Eq. (17). These constants can be used therefore to give linear expressions for the functions $H_{0}(r)$ and $\delta U(r)$ near the surface:

$$
H_{0}(r)=H_{0}(R)-(R-r) H_{0}^{\prime}(R)+\mathcal{O}(R-r)^{1+\tau},
$$

$$
\delta U(r)=\delta U(R)-(R-r) \delta U^{\prime}(R)+\mathcal{O}(R-r)^{1+\tau},
$$

where $0<\tau \leq 1$ is a positive constant that depends on the equation of state. For soft equations of state $1<\gamma<$ 2 the singularities are relatively beneign, and the second derivatives $H_{0}^{\prime \prime}$ and $\delta U^{\prime \prime}$ are finite at $r=R$. Thus, in this case series expansions analogous to Eqs. (B7) and (B8) can be obtained for the derivatives:

$$
\begin{gathered}
H_{0}^{\prime}(r)=H_{0}^{\prime}(R)-(R-r) H_{0}^{\prime \prime}(R)+\mathcal{O}(R-r)^{2}, \\
\delta U^{\prime}(r)=\delta U^{\prime}(R)-(R-r) \delta U^{\prime \prime}(R)+\mathcal{O}(R-r)^{2},
\end{gathered}
$$

where the constants $H_{0}^{\prime \prime}(R)$ and $\delta U^{\prime \prime}(R)$ are evaluated directly from the Eqs. (11) and (12). In the stiff case, $\gamma>$ 2, series expansions can also be found using Eqs. (B4)(B6) and the equations (11)-(12). These expansions are not analytic at $r=R$ but can nevertheless be used to approximate the derivatives $H_{0}^{\prime}(r)$ and $\delta U^{\prime}(r)$ near the surface:

$$
\begin{array}{r}
H_{0}^{\prime}(r)=H_{0}^{\prime}(R)-\frac{4 \pi}{M} R^{2} \rho(r)\left[2 \delta U(R)-H_{0}(R)\right] \\
\times[1+\mathcal{O}(R-r)],
\end{array}
$$

$$
\begin{aligned}
& \delta U^{\prime}(r)=\delta U^{\prime}(R) \\
& \quad-\frac{1}{2}\left\{\frac{4 \pi}{M} R^{2}\left[1-\beta_{1}(R)\right] \rho(r)\left[2 \delta U(R)-H_{0}(R)\right]\right. \\
& \left.\quad+\beta_{3}(r) \delta U^{\prime}(R)+\beta_{4}(r) \delta U(R)\right\}[1+\mathcal{O}(R-r)] .
\end{aligned}
$$

[1] K. S. Thorne, Astrophys. J. 158, 1 (1969).

[2] L. Lindblom and S. L. Detweiler, Astrophys. J. Suppl. 53, 73 (1983).

[3] S. Chandrasekhar and V. Ferrari, Proc. Roy. Soc. (London) A 432, 247 (1991).

[4] K. D. Kokkotas and B. F. Schutz, Mon. Not. Roy. Astro. Soc. 255, 119 (1992).

[5] J. R. Ipser and L. Lindblom, Astrophys. J. 355, 226 (1990).

[6] C. Cutler and L. Lindblom, Astrophys. J. 385, 630 (1992).

[7] K. S. Thorne and A. Campolattaro, Astrophys. J. 149, 591 (1967) and K. S. Thorne and A. Campolattaro, Astrophys. J. 152, 673 (1967).

[8] J. R. Ipser and L. Lindblom, Astrophys. J. 389, 392 (1992).

[9] S. Detweiler and L. Lindblom, Astrophys. J. 292, 12 (1985).

[10] J. R. Ipser and R. H. Price, Phys. Rev. D 43, 1768 (1991).

[11] L. Lindblom and R. J. Splinter, Astrophys. J. 348, 198, (1990)

[12] J. L. Friedman and B. F. Schutz, Astrophys. J. 200, 204 (1975). 
[13] The function $h(r)$ vanishes linearly at the surface of any equilibrium stellar model. This makes $h(r)$ the most slowly vanishing themodynamic function at the stellar suface having a finite gradient there. Imposing the boundary condition $\Delta h=0$ at the surface of the star insures that the Lagrangian changes in other more rapidly vanishing potentials (such as the pressure) will also vanish there.

[14] K. S. Thorne, Astrophys. J. 158, 997 (1969).

[15] J. Ipser, Astrophys. J. 166, 175 (1971).

[16] N. Stergioulas, Ph. D. Thesis, University of Wisconsin at Milwaukee (1996); N. Stergioulas and J. L. Friedman, gr-qc/9705056.

[17] Unfortunately the $M / r$ dependence of the exact expression for $H_{0}$ is a very slowly converging series in $M / r$. Adding the first order $M / r$ correction to the imaginary part of Eq. (28) can be accomplished by multiplying that term by $1-l M / r$. This correction reduces the accuracy of the frequencies of the modes in the most relativistic models. Presumably a more accurate boundary condition than Eq. (28) could be constructed by adding the appropriate higher-order terms in $M / r$.

[18] H. A. Bethe and M. Johnson, Nucl. Phys. A 230, 1 (1974).

TABLE I. The exact frequencies of the $f$-modes of $1.4 M_{\odot}$ stellar models are compared with those computed using the near-zone boundary condition, and with those computed using several other approximation methods.

\begin{tabular}{lcccccc}
\hline \hline & $l$ & Cowling & post-Newtonian & $\begin{array}{c}\text { Lowest-Order } \\
\text { Near-Zone }\end{array}$ & $\begin{array}{c}\text { Higher-Order } \\
\text { Near-Zone }\end{array}$ & Exact \\
\hline $\operatorname{Re}(\omega)$ & 2 & 1.4484 & 1.231 & 1.1672 & 1.2018 & 1.2016 \\
$\operatorname{Re}(\omega)$ & 3 & 1.7469 & 1.619 & 1.5716 & 1.5863 & 1.5862 \\
$\operatorname{Re}(\omega)$ & 4 & 1.9906 & 1.907 & 1.8652 & 1.8739 & 1.8738 \\
$\operatorname{Re}(\omega)$ & 5 & 2.2031 & 2.147 & 2.1071 & 2.1130 & 2.1129 \\
$\operatorname{Im}(\omega)$ & 2 & - & - & $5.60 \times 10^{-4}$ & $3.53 \times 10^{-4}$ & $3.95 \times 10^{-4}$ \\
$\operatorname{Im}(\omega)$ & 3 & - & - & $7.29 \times 10^{-6}$ & $3.56 \times 10^{-6}$ & $3.96 \times 10^{-6}$ \\
$\operatorname{Im}(\omega)$ & 4 & - & - & $9.36 \times 10^{-8}$ & $3.78 \times 10^{-8}$ & $4.01 \times 10^{-8}$ \\
$\operatorname{Im}(\omega)$ & 5 & - & - & $1.09 \times 10^{-9}$ & $3.70 \times 10^{-10}$ & $3.63 \times 10^{-10}$ \\
\hline \hline
\end{tabular}

